\title{
New Trends to Support Independence in Persons with Mild Dementia - A Mini-Review
}

\author{
M. Mokhtaria, d H. Aloulou ${ }^{a} \quad$ T. Tiberghien ${ }^{a} \quad$ J. Biswas ${ }^{a, b} \quad$ D. Racoceanu ${ }^{a} \quad$ P. Yap ${ }^{c}$

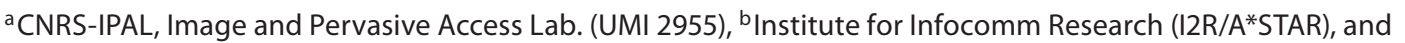 \\ ${ }^{c}$ Khoo Teck Puat Hospital, Singapore, Singapore; ${ }^{d}$ Institut Mines-Telecom, Paris, France
}

\section{Key Words}

Smart homes $\cdot$ Human-machine interaction $\cdot$ Cognitively impaired individuals $\cdot$ Context awareness $\cdot$ Reasoning engine

\begin{abstract}
Our research was motivated by the growing aging population worldwide and the need to concentrate research efforts on a specific target group; it focuses on elderly persons with physical and cognitive deficiencies. The primary goal is to enable persons with mild dementia to maximize their physical and mental functions through assistive technologies in order to be able to continue to participate in social networks and lead independent and purposeful lives. Persons with mild dementia usually have problems in performing activities of daily living due to episodic memory decline. These can include simple activities, such as bathing, changing clothes and preparing meals. Through extended field test trials involving end users, we have demonstrated that assistive technology that provides timely prompts, alarms and reminders can enable them to preserve their abilities and improve their quality of life. Understanding the user context, especially when targeting demented individuals, and providing the required personalized assistive services is the objective of our research work. Finding the appropriate user
\end{abstract}

interface to interact with the provided services is often a barrier. Thus, we have adopted the approach of a multimodal interactive system with the living environment including a TV set, iPad-like tablets, sensors/actuators, and wireless speakers connected to a reasoning engine that is able to consider the complexity of the users' profile defined by his/ her cognitive abilities. In this paper we will mainly focus on the interaction level with the system as well as on the validation stages performed to meet the users' requirements. This is the result of several years' work since 2006 in the frame of two projects (IST-FP6 COGKNOW European completed project and AMUPADH ongoing project in Singapore).

Copyright $\odot 2012$ S. Karger AG, Basel

\section{Introduction}

Population aging and the lack of caregivers to assist older people bring about a problem of increasing significance in different regions of the world. In 1995, it was estimated that 371 million people ( $6 \%$ of the world's population) were over the age of 65 . This proportion is much higher in Europe (14\%) and North America (13\%) than in Latin America and Asia (5\%) or Africa (3\%) [1]. The European Commission has raised awareness on this demographic evolution as illustrated in figure 1.

\section{KARGER}

Fax +41613061234

E-Mail karger@karger.ch

www.karger.com
(C) 2012 S. Karger AG, Basel

0304-324X/12/0586-0554\$38.00/0

Accessible online at:

www.karger.com/ger
Dr. Mounir Mokhtari

CNRS-IPAL, Image and Pervasive Access Lab. (UMI 2955)

1 Fusionopolis Way, \#21-01 Connexis (South Tower)

Singapore 138632 (Singapore)

Tel. +65 6408 2547, E-Mail mounir.mokhtari@it-sudparis.eu 
Fig. 1. European demographic evolution (more than 65 years old in millions).

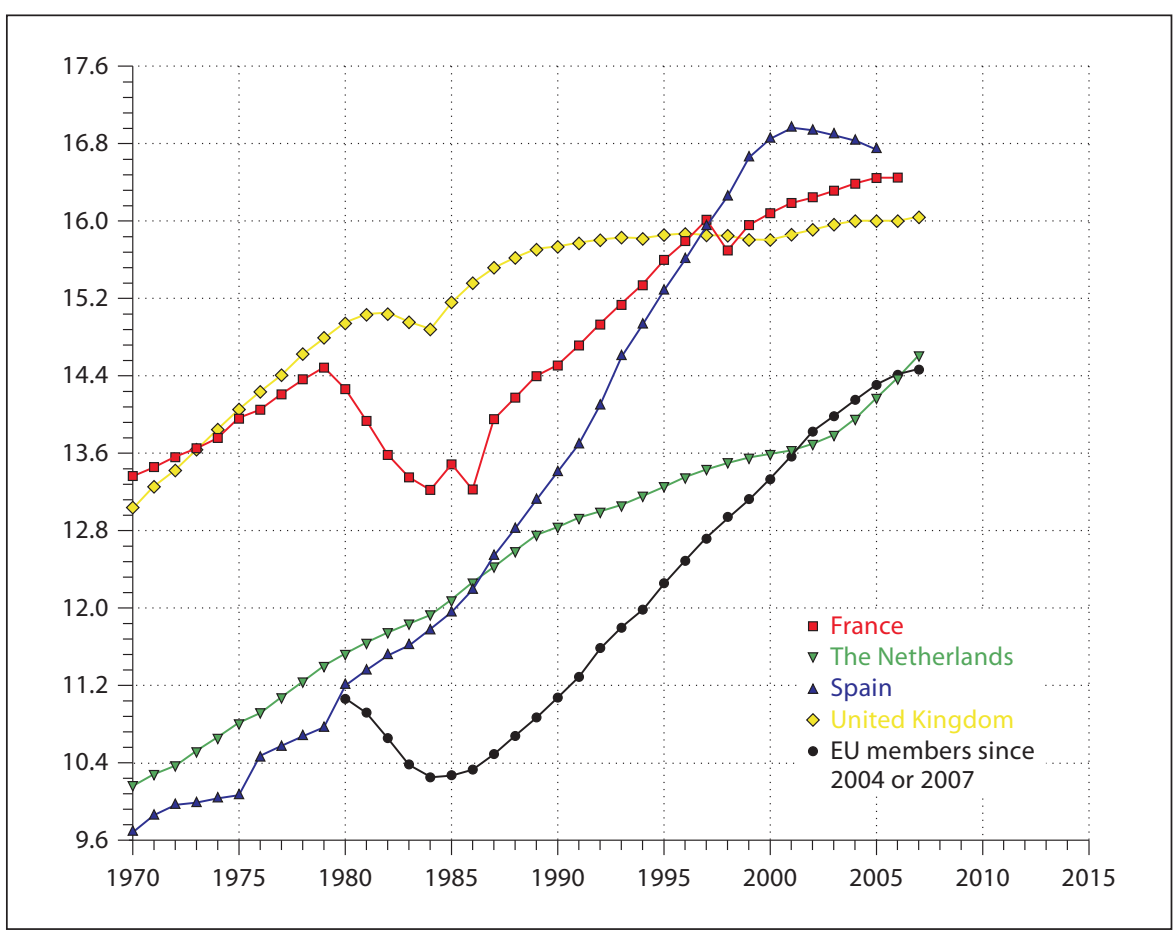

Elderly people may suffer from different chronic diseases and need special care that entails high expenditures. Dementia is one of the most important chronic diseases. The World Alzheimer Report 2009 report [2] released for World Alzheimer's Day (2009) indicates that 35 million people are suffering from dementia and estimates that the number of cases will double every 20 years. During the next 20 years, this number will increase by $40 \%$ in Europe, $63 \%$ in North America, $77 \%$ in the Southern Cone of Latin America and 89\% in the developed AsiaPacific countries. These figures are to be compared with the 117\% growth in East Asia, 107\% in South Asia, 134$146 \%$ in the rest of Latin America, and 125\% in North Africa and the Middle East.

Dementia is a progressive, disabling, chronic disease affecting $5 \%$ of all people above 65 and over $40 \%$ of people over 85 years. The cost of care and assistance for this disease is getting very high; the World Alzheimer Report 2010 economic report [3] estimates that the worldwide cost of dementia is USD 604 billions a year. This is why it is urgent to focus on this category of older people and try to adapt the intelligent home to their needs.

This situation has motivated computer researchers to look for solutions to solve the problem, taking advantage of the developments in information technologies and tools for identification and monitoring. The idea is to de- sign intelligent systems that are able to support demented persons in performing their activities of daily living, and to provide all commodities necessary to maintain/increase their autonomy. The guiding principle is to provide remote monitoring of demented persons as they carry out their daily tasks and to intervene when appropriate.

\section{Level of Intervention}

Dementia is characterized by progressive deterioration of intellectual and functional abilities, typically over a period of 7-10 years [4] and is classified into 5 stages (3-7) according to the Global Deterioration Scale [5]. These stages are described in table 1.

At stage 3, the symptoms can be subtle and the patient can live independently without assistance [6]. At stages 4 and 5, independent living becomes an issue, and in more advanced stages of the disease, the situation becomes critical, especially with verbal communication problems (aphasia), difficulty in identifying objects and persons (agnosia), and high-level disorder in performing familiar and learned tasks (apraxia). The patient, therefore, needs continuous support by a professional caregiver because he or she can no longer perform everyday tasks. 
Table 1. The global deterioration scale for assessment of primary degenerative dementia

\begin{tabular}{|c|c|c|}
\hline \multicolumn{2}{|c|}{ Level } & \multirow{2}{*}{$\begin{array}{l}\text { Clinical characteristics } \\
\text { No subjective complaints of memory deficit. No memory deficit evident on clinical interview. }\end{array}$} \\
\hline 1 & No cognitive decline & \\
\hline
\end{tabular}

4 Moderate cognitive decline (mild dementia)

Clear-cut deficit on careful clinical interview. Deficit manifest in the following areas:

(a) decreased knowledge of current and recent events; (b) may exhibit some deficit in memory of one's personal history; (c) concentration deficit elicited on serial subtractions; (d) decreased ability to travel, handle finances. Frequently no deficit in following areas: (a) orientation to time and place; (b) recognition of familiar persons and faces; (c) ability to travel to familiar locations. Inability to perform complex tasks. Denial is the dominant defense mechanism. Flattening of affect and withdrawal from challenging situations frequently occur.

5 Moderately severe cognitive decline (moderate dementia)
Patient may no longer survive without assistance. Patient is unable to recall a major relevant aspect of their current lives during an interview, e.g. an address or telephone number of many years, the names of close family members (such as grandchildren), the name of the high school or college from which they graduated. Frequently, some disorientation to time (date, day of week, season, etc.) or to place. An educated person may have difficulty counting back from 40 by $4 \mathrm{~s}$ or from 20 by $2 \mathrm{~s}$. Persons at this stage retain knowledge of many major facts regarding themselves and others. They invariably know their own names and generally know their spouse's and children's names. They require no assistance with toileting and eating, but may have some difficulty choosing the proper clothing to wear.

May occasionally forget the name of the spouse upon whom they are entirely dependent for survival. Will be largely unaware of all recent events and experiences in their lives. Retain some knowledge of their past lives but this is very sketchy. Generally unaware of their surroundings, the year, the season, etc. May have difficulty counting from 10 both backward and, sometimes, forward. Will require some assistance with activities of daily living, e.g. may become incontinent, will require travel assistance but occasionally will be able to travel to familiar locations. Diurnal rhythm frequently disturbed. Almost always recall their own name. Frequently, continue to be able to distinguish familiar from unfamiliar persons in their environment. Personality and emotional changes occur. These are quite variable and include: (a) delusional behavior, e.g. patients may accuse their spouse of being an impostor, may talk of imaginary figures in the environment, or to their own reflection in the mirror; (b) obsessive symptoms, e.g. person may continually repeat simple cleaning activities; (c) anxiety symptoms, agitation and even previously nonexistent violent behavior may occur; (d) cognitive abulia, i.e. loss of willpower because the individual cannot carry the thought long enough to determine a purposeful course of action.

All verbal abilities are lost over the course of this stage. Frequently there is no speech at all only unintelligible utterances and rare emergence of seemingly forgotten words and phrases. Incontinent of urine, requires assistance toileting and feeding. Basic psychomotor skills, e.g. ability to walk, are lost with the progression of this stage. The brain appears to no longer be able to tell the body what to do. Generalized rigidity and developmental neurologic reflexes are frequently present. 
Our strategy is to focus on stages 3-5 of the disease, at which the deficits are typically still mild and, therefore, amenable to assistive intervention. At these stages, the patient experiences difficulties in planning, organizing and sequencing that prevent him/her from performing tasks in an ordered and sequenced manner. Distractions (a phone call or an unusual sound) or a short-term memory problem may lead the patient to skip steps unwittingly or to perform actions that are unrelated to his or her original goal [7]. Monitoring and intervention when necessary can help the patient remain independent in his or her home for as long as possible [8].

\section{Elicitation of User Needs in Terms of Technological Assistance}

Technology through assistive devices aims to enable aging in place. Based on several years of investigation on user needs and analysis of elderly people with cognitive decline, through the EU COGKNOW project [9], we were able to summarize the top 4 requirements as follows:

- remembering to perform simple daily living tasks, such as preparing meals, bathing, grooming;

- maintaining their social links;

- feeling motivated to participate in everyday life, and

- boosting their feeling of safety.

We have performed our own user investigation in our laboratory in France in the frame of a $\mathrm{PhD}$ thesis on the use and impact of information and communication technology in collaboration with Sherbrooke University in Canada [10]. The objective was to focus on defining assistive services and analyzing the human-machine interaction with smart environment. Thirty-five cognitively impaired persons participated in the study. This allowed us to design the technological assistive environment described in this paper taking into account the needs, capabilities and sociological aspects of elderly people in their living environment.

Elderly people are usually not comfortable with computers or even mobile devices and this applies even more for demented persons. Through multiple experimentations involving end users, we came to the conclusion that in the home environment a TV set is better suited to provide assistive services than a computer. Four main categories of TV services have been identified (fig. 2):

- information: providing reminders and managing the user's agenda;

New Trends to Support Independence in

Persons with Mild Dementia
- communication: maintaining the social link with relatives and friends;

- action: controlling home devices with less effort (doors, windows or lights)

- leisure: playing games, listening to music or watching a video on demand.

\section{Context-Aware System}

Context awareness is the capacity of a system to take into account the users' present status and activity plus all the information surrounding the user, including user characteristics, when providing services to him/her. Given this approach, context-aware systems can offer services that bring about a relevant and significant improvement in his/her daily life.

Context management can be defined as the process enabling context awareness. This process comprises different subtopics, in particular context acquisition and context reasoning. Context acquisition is the starting point of the mechanism. It relies on the deployment of devices and sensors in the environment, and also on wearable (on body) sensors and devices carried by the user, such as mobile phones, which allow implicit and explicit interaction between the user and the system. A reasoning module then processes this information. Depending on the kind of services the system provides, the reasoning process will decide on the specific situations and provide the corresponding service, for example in the form of a reminder.

In the European COGKNOW project [11], which is one of the first projects dealing with technology supporting people with mild dementia in Europe, we notably focused on reminder functionalities. The reasoning is traditionally based on a predefined plan of activities the person has to complete during the day and a recall service is provided when necessary. The reasoning will then combine temporal information with various contextual clues from which it will infer whether the activity has not been completed successfully or satisfactorily and whether a recall is necessary.

As a conflicting situation, the person may receive a phone call. While speaking on the phone, he or she may miss a reminder, or forget it. In this case, there is a conflict between the preplanned activity and the real activity. The context-aware system must have the ability to manage this kind of situation. The proposed solution and the following implementation are presented in this paper.

Gerontology 2012;58:554-563 


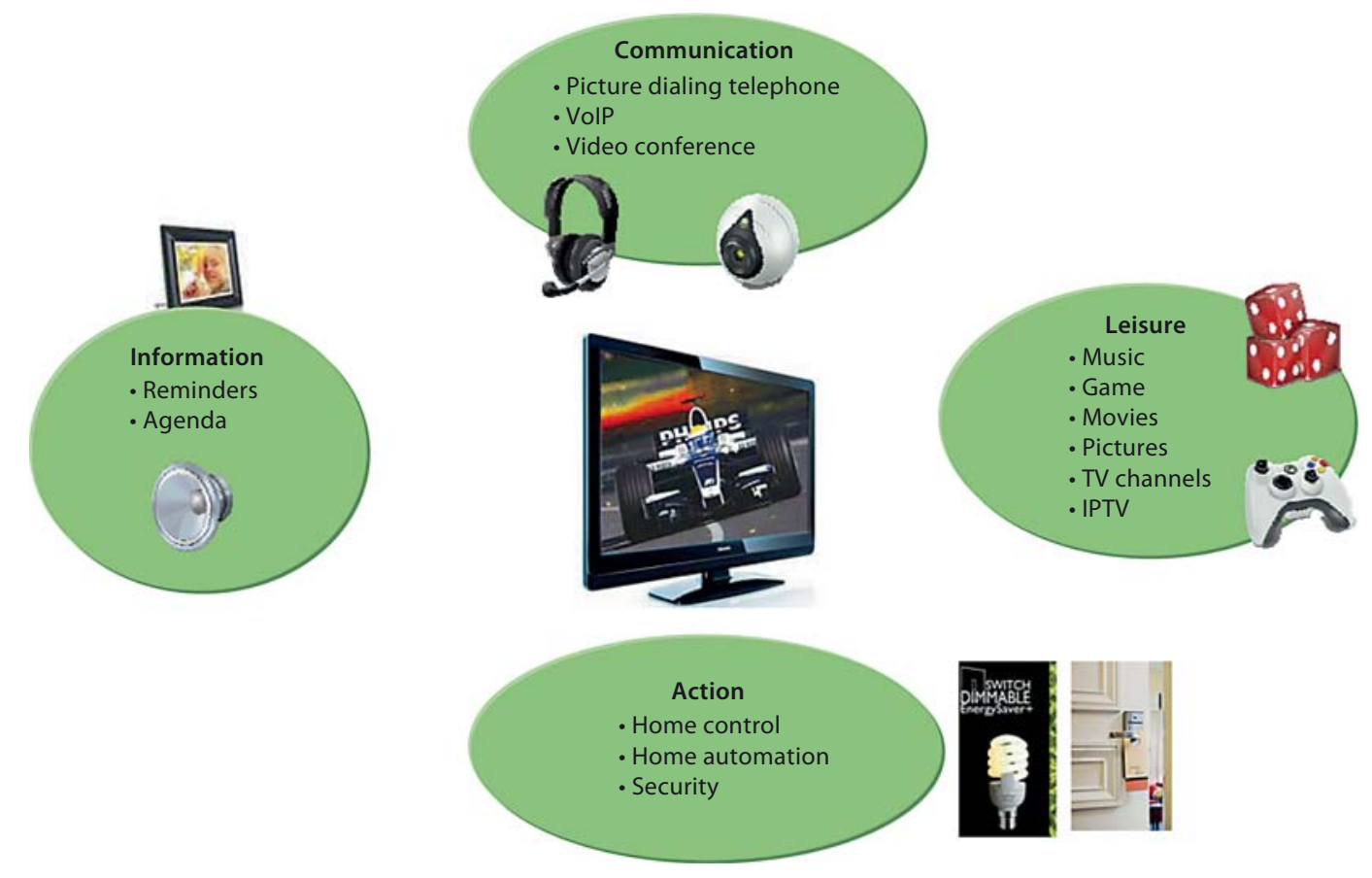

Fig. 2. Interactive TV system to support aging people with dementia. VoIP = Voice over Internet Protocol.

\section{Conflict Management Scenarios and Design}

When defining relevant disruptive activities, we have only focused on the most frequent ones that are both critical and can be tracked by the context-aware system. We have identified two key disruptive activities as follows:

- answering telephone calls and

- sleeping

The main reason for choosing these two activities is that both are common daily activities. When the demented person performs these activities, it is not appropriate for the reminding system to simply interrupt him or her with a prompt to execute the preplanned activity, which may irritate the user. Other disruptive activities, such as someone knocking at the door, food cooked being ready or a fire alarm, could be easily managed using different types of sensors.

In order to observe whether the user is engaged in any of the disruptive activities, various ubiquitous sensors are required to track the context of the user's activities. As a first step, we focused more on usability, by using off-theshelf sensors with approximate information, than on the quality of sophisticated sensors.

A simple contact sensor attached to the telephone handset can easily detect the context of answering a telephone call. If the sensor detects that the handset is picked up, we just assume that the user is answering a phone call. Recently, we have added a shake sensor and a microphone as ambient sensors which could calibrate more precisely such activity. The context of sleeping can be reasoned by the following rule ${ }^{1}$ :

(oldPerson, locatedIn, bed) $\wedge$ (bedroom, lightStatus, OFF) $\wedge$ (bedroom, drapStatus, CLOSED) $\rightarrow$ (oldPerson, situation, SLEEPING)

1 Rules description uses the RDF (Resource Description Framework) triplet notation: (source, source's property, property's values). 
Fig. 3. Architecture of the Context Engine

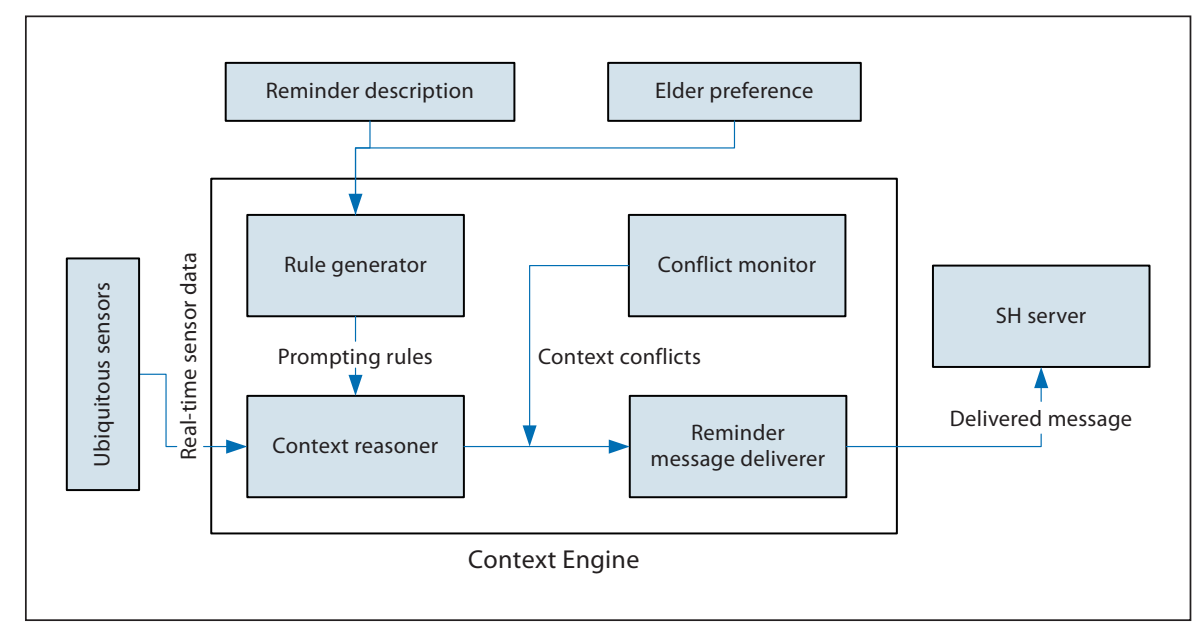
module.

This explicit rule has some limitation, for example if the user is having a nap during the day in a reclining chair in the living room rather than in the bed, which is a frequent situation, than this rule will not be fired. For this reason, we have designed an ontological model representing a classification of activities of daily living according to each user. The context engine will then recognize the user contextual situation (context acquisition) according to activities being performed (activity monitoring). This part will not be described in this paper.

We have introduced three conflict management scenarios around these two disruptive activities. In each case, we easily identify the preplanned activity, the disruptive one and the prompting strategy that follows:

Scenario 1: It is almost time to deliver a reminder (such as Lunch Reminder), and the user is on the phone. The reminder will be delayed until the phone call is terminated. To confirm that the call was terminated, as a switch sensor is not sufficient in case the user forgets to replace the receiver, we are actually considering a shake sensor attached to the receiver to detect the hand movement.

Scenario 2: When the user is preparing the meal (lunch or dinner), he/she is interrupted by a phone call, and does not come back to continue preparing the meal, the system will deliver a reminder to prompt him/her to resume the meal preparation.

Scenario 3: When it is nearly time to deliver a reminder (e.g. to watch TV) and the user is sleeping, the reminder will be delayed until the user wakes up. If the time for the reminder expires, the reminder will be automatically canceled. Otherwise, it will be delivered after the user wakes up.

New Trends to Support Independence in Persons with Mild Dementia
The architecture of the Context Engine is shown in figure 3. The Context Engine acquires reminder configuration from both caregivers and the user, and generates prompting rules for each reminder. The prompting rule contains two basic pieces of information for each reminder: the time schedule and the way to handle context conflicts according to the user's preference. When the Context Engine decides to deliver a reminder, it will send messages to trigger the prompting on the Smart Home Server (SH Server). Then, the SH Server analyzes the context information to decide which device should be used to display the reminder. This adaptation of the interaction to the context is part of the so-called User Interface Plasticity that will be described further in this paper.

Several states were developed within the Context Engine module to allow the context reasoner to call these states under its own control:

- cancel a reminder;

- delay a reminder specifying a new time;

- advance a reminder specifying a new time;

- prompt a message;

- interrupt a current message, and

- cancel a delayed message

\section{User Interface Plasticity}

The term 'plasticity' is inspired from the property of materials that expand and contract under natural constraints without breaking, thus preserving continuous usage [12]. Applied to Human Machine Interface, plasticity is the capacity of an interactive system to withstand variations in context of use while preserving us- 
ability. It proposes an answer in smart spaces to the diversity of environments, activities, displays and devices people can be surrounded with. This enables a more natural and seamless kind of interaction that will help demented users to engage in interacting with computational services, which they might not be used to. For us, this concept is composed of two main aspects: multimodality of access to the services, which corresponds to the selection of the interaction device depending on the environment and context; and polymorphism, which corresponds to the adaptation of the user interface to the interactive device.

In terms of multimodality, context information coupled with a reasoner is able to decide autonomously which modality of interaction (or simply the device) needs to be used to display the reminders. Depending on the location and the activity of the older person, some devices might be a better choice. For example, if he/she is in the living room watching $\mathrm{TV}$, we should send the reminder there, but if he/she is reading a book or taking a shower, nearby speakers would be a more relevant choice. The polymorphism aspect is implemented to perform real-time generation of the reminder interface depending on user profile, device of use and context. For example, the design of the interface should comply with the user's preferences and deficiencies as well as with the device characteristics. As an adaptation to the context, we can plan that the volume of an audio reminder will be adjusted at night if several users share the same room.

As the reasoner selects the device displaying the reminder, it can influence who is receiving it. Consequently, it enables alerts to caregivers (nurses, family members, neighbors) as we can program the system to send the reminder to a given mobile phone in critical situations. For example, if we reminded the user several times to turn off the gas after cooking and he/she still ignores the reminder, we might decide to escalate the reminder into an alert to a nearby caregiver. This enhances the user's safety and independence.

\section{System Integration and Experimentation}

Figure 4 shows some examples of cost-effective sensors. Our idea was to use simple sensors available on the market in order to facilitate the deployment of the system in users' homes using an accepted and reliable system: figure $4 \mathrm{a}$ is a simple contact sensor which is used to detect whether the handset of the telephone is lifted (is off hook) and hence can assume, with a certain confidence,

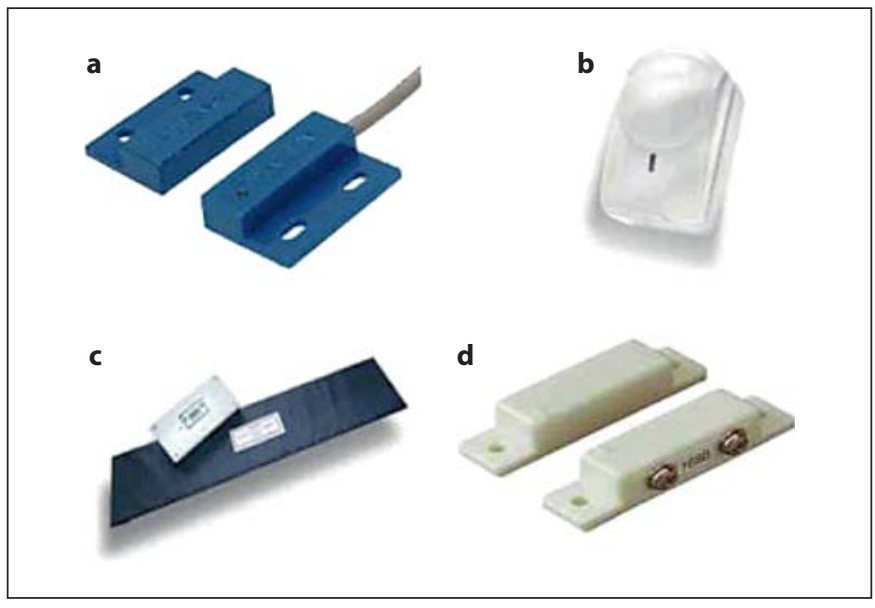

Fig. 4. Ubiquitous sensor used. a Contact sensor. b Motion sensor. c In-bed sensor. d Door sensor.

whether the user is on the phone or not (depending on the user's profile). Figure $4 \mathrm{~b}$ is the motion sensor, which is used for activity detection. It is a passive infrared sensor embedded with an integral $169.48125-\mathrm{MHz}$ transmitter. Figure $4 \mathrm{c}$ is the in-bed sensor for bed occupancy detection. It is placed between the bed base and the mattress. The in-bed sensor has 4 levels of sensitivity, which can be configured for each user. It is embedded with integral 169.48125- and 433.92-MHz transmitters. Figure $4 \mathrm{~d}$ is the door sensor used to detect whether the door is open or closed.

An interactive TV system has been developed and presented during the Techfest 2009 exhibition organized by the Institute for Infocomm Research (I2R/A*STAR) in October 2009.

The system is composed of an Internet Protocol television (IPTV) system that is able to handle external ubiquitous sensor events and to control the different functionalities with a Wiimote remote control using integrated accelerometers/gyroscopes and infrared transceivers to define the hand/cursor position. We are adding the accelerometer information available in the Wiimote. The idea was to couple the hand movement through the Wiimote to select the corresponding interactive menus/function displayed on the TV. Communication between Wiimote and IPTV box is performed through a Bluetooth wireless protocol. Standard TV remote controllers can also be used if needed through an implemented infrared receiver (fig. 5).

The displayed reminders are adapted to each user profile (stored on a USB key). Texts, images and videos can be 


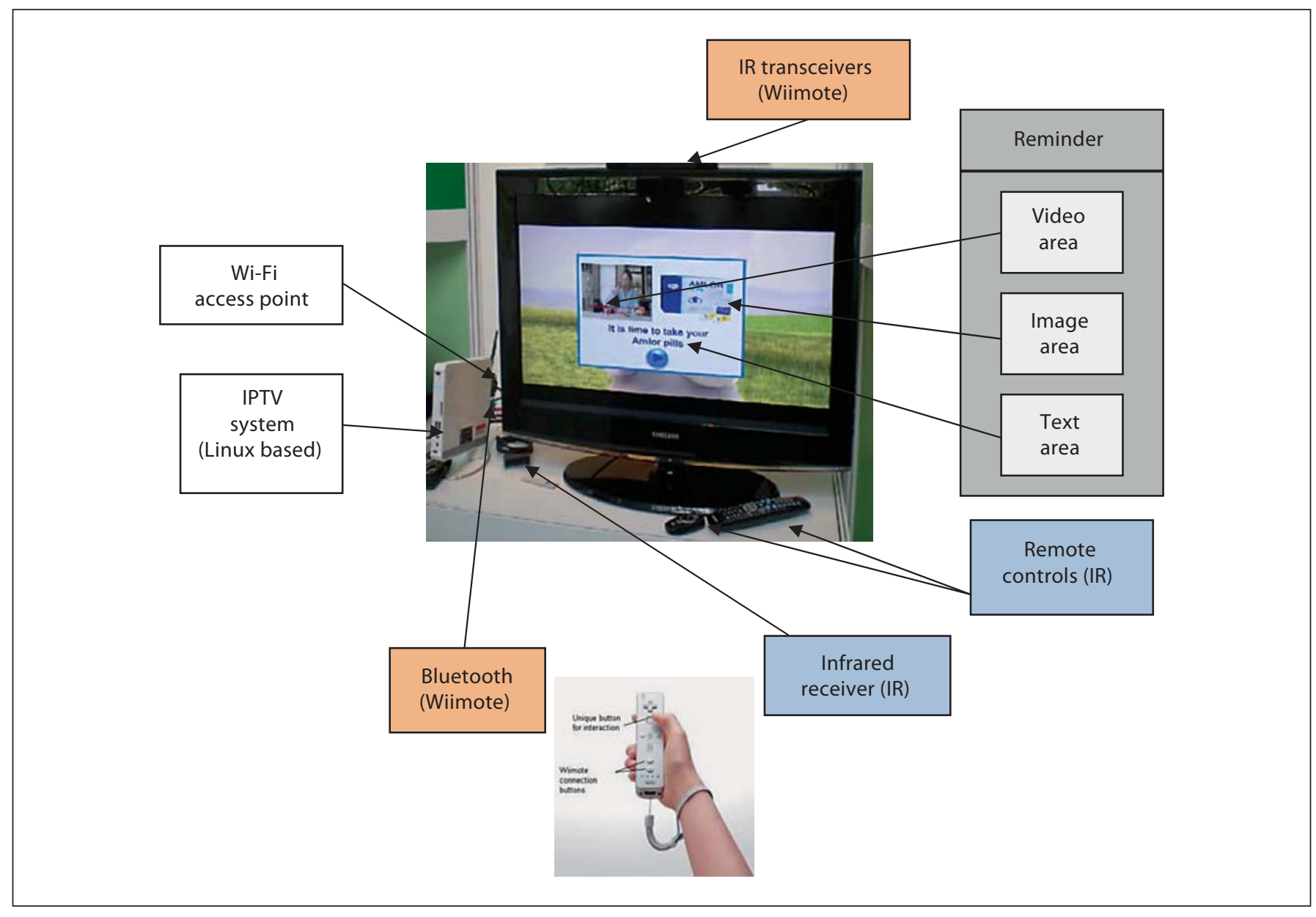

Fig. 5. Demonstration of the system at Techfest 2009, Singapore.

combined to build the reminding information. Wi-Fi access points are used in order to retrieve the users' personalized content and data from any server (Web services).

Several menus have been implemented as shown in figure 6 .

The upgraded version of the system presented at Techfest 2010 integrates the implementation of User Interface Plasticity and the escalation of reminders toward alerts. For this edition at the exhibition, we demonstrated a smart space made up of two environments: a living room area with the interactive TV system described previously and a kitchen area that had a tablet PC with a similar interactive system. Depending on the the user's location and activity, reminders 'followed' them to increase their impact and their attitude towards the interactive platform. Reminders were sent to the TV while the user was watching it, to the tablet PC when his/her presence was detected

New Trends to Support Independence in Persons with Mild Dementia in the kitchen and via Bluetooth to speakers in any other case. Speakers are interesting here due to their omnipresence in the environment using the user's background awareness; it represents a reliable mode of interaction. Concerning the escalation of reminders into alerts, we implemented mobile platforms running on iPhone and Android smart-phones targeting a use by caregivers. These platforms are connected to our Smart Home Server to receive alerts when needed. The phone will then vibrate and display a reminder similar to the one shown in figure 7.

\section{Conclusion}

The experience gained from developing a series of situation-aware services for elderly people without cognitive decline (NUADU project) [13] and demented people 

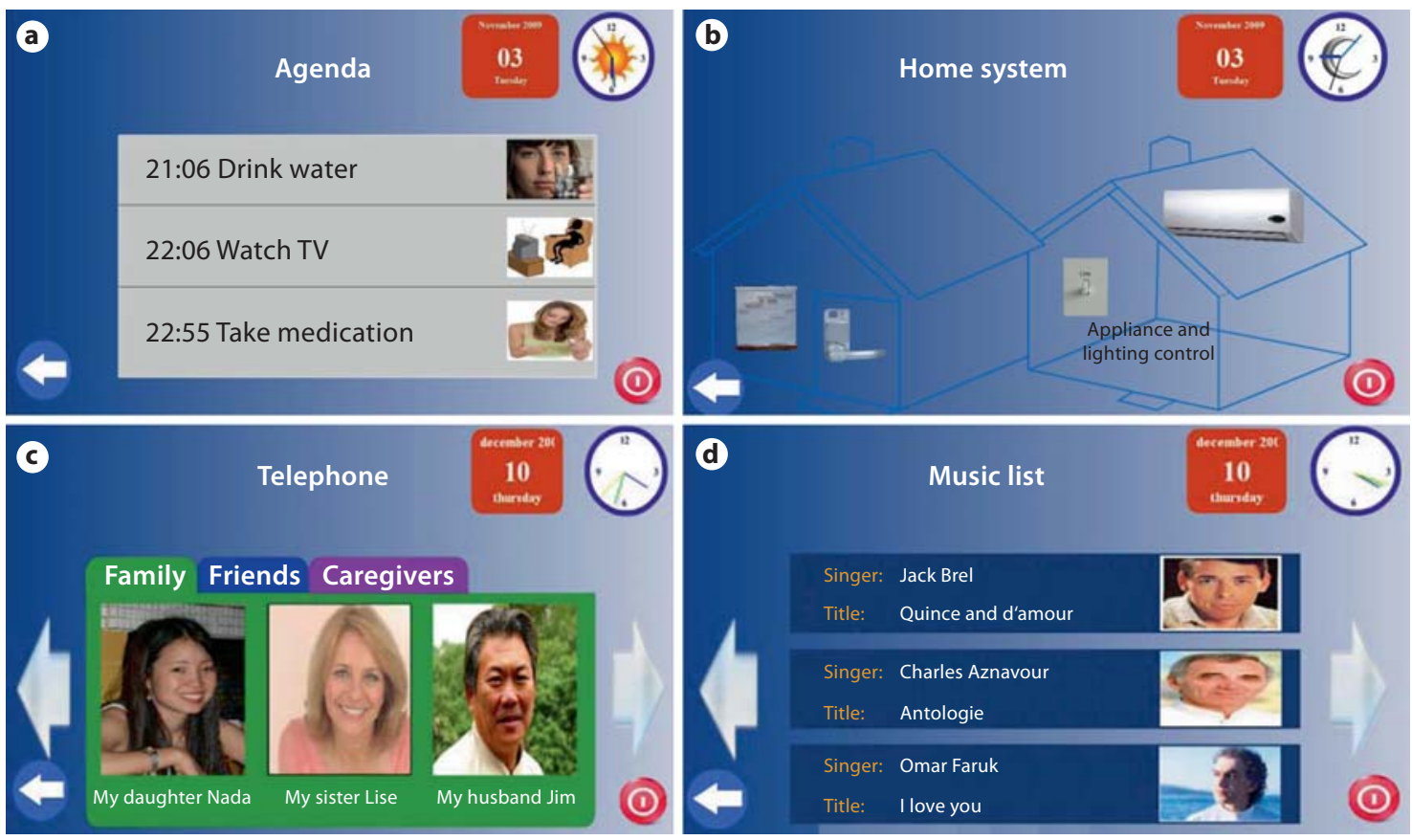

Fig. 6. Interactive TV menus. a Agenda. b Home control. c Picture dialing telephone. d Music.

(COGKNOW project) has provided valuable insights into the challenges and opportunities within this area in Europe. As such, the following points have been considered as the lessons learnt and hence provided some areas for consideration for the ongoing AMUPADH project [14].

Engineering vision in designing technological system does not always meet user's requirements. During our investigation on both the users' side, involving representative end-users and users in several projects and through a PhD thesis performed in our lab, we have highlighted the fact that human-machine interaction is a critical issue. To tackle with this issue, we have adopted a pilot site approach which consists in deploying a technological system, out of the research lab, in the living environment of users by establishing several pilot sites in Europe and one in Singapore at Peacehaven Nursing Home. As such, the requirement to collect data from real users of the system over long periods of time became essential for validation purposes.

We have now received approval from the Local Ethical Committee in Singapore and started an experiment involving two residents of Peacehaven Nursing Home in close collaboration with Alexandra Hospital. The trials started in October 2011, after completion of the deploy-
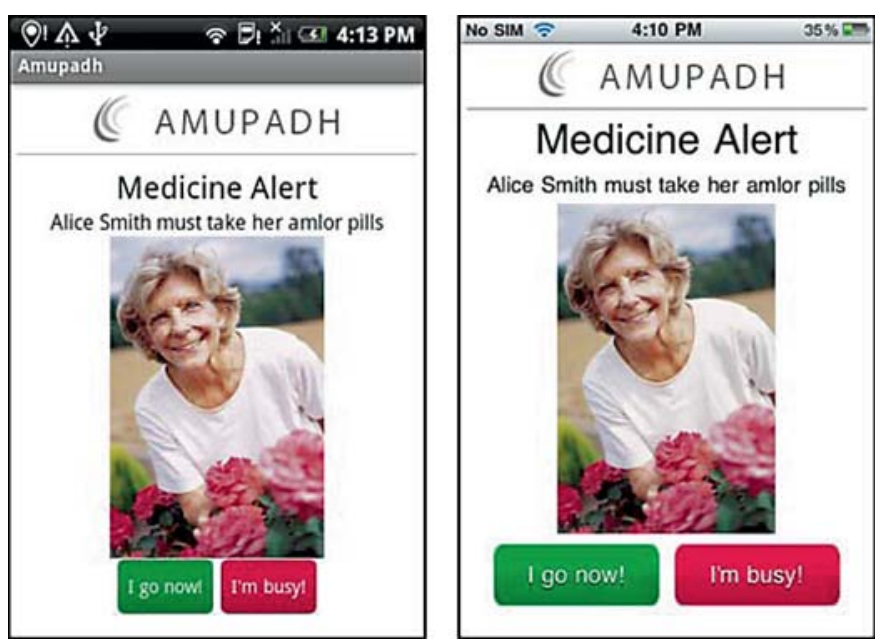

Fig. 7. Alerts on Android (left) and iPhone (right) mobile platforms.

ment of the integrated AMUPADH system in the nursing home. Data acquisition and analysis are ongoing. Detailed use impact and evaluation methodology will be provided in a forthcoming publication due to be ready by the end of 2012. 


\section{References}

1 Alzheimer's Disease International: The demography of ageing around the world. Factsheet 4. http://www.alz.co.uk/adi/pdf/ 4demog.pdf).

2 Prince M, Jackson J: World Alzheimer Report 2009, Alzheimer's Disease International. http://www.alz.co.uk/research/files/ WorldAlzheimerReport.pdf.

3 World Alzheimer Report 2010:http://www. alz.co.uk/research/files/World AlzheimerReport2010ExecutiveSummary.pdf.

4 Diamond J: A report on Alzheimer disease and current research. Technical report. Toronto, Alzheimer Society of Canada, October 2005.

5 Reisberg B, Ferris SH, Leon MJ, Crook T: The Global Deterioration Scale for assessment of primary degenerative dementia. Am J Psychiatry 1982;139:1136-1139.

6 Pigot H, Mayers A, Giroux S: The intelligent habitat and everyday life activity support. Proc 5th Int Conf Stimulations Biomed, Ljubljana, 2003, pp 507-516.
7 Patterson DJ, Etzioni O, Fox D, Kautz H: Intelligent Alzheimer's patients: enabling the cognitively disabled; in Proc 1st Int Works on Ubiquitous Computing for Cognitive Aids, Göteborg, 2002.

8 Boger J, Poupart P, Hoey J, Boutilier C, Fernie G, Mihailidis A: A decision-theoretic approach to task assistance for persons with dementia. Proc 19th Int Jt Conf Artif Intelligence (IJCAI05), Edinburgh, 2005, pp 1293-1299.

9 COGKNOW Final Evaluation report D.5.7.1., M. Dröes, J.E. Bengtsson (eds): http://www.cogknow.eu/1/FP6_COGKNOW/system/files/DEL5.7.1-Novay-FinalEvaluationReport-v3.0.pdf.

10 Arab F, Pigot H, Rabardel P, Folcher V, Rigaud AS, Mokhtari M: Age, memory and time: practices and support. J Assoc Adv Model Stimul Tech Enterprises (AMSE) 2011;71:136-144.
11 'COGKNOW', September 2006 to August 2009, http://www.cogknow.eu/1/FP6_COGKNOW/news/cogknow-project-start-date. html.

12 Thevenin D, Coutaz J: Plasticity of user interfaces: framework and research agenda. Proc IFIP TC $13^{\text {th }}$ Conf Hum Comput Interact, Interact'99, Edinburgh, 1999. Amsterdam, IOS Press, 1999.

13 'NUADU', April 2006 to April 2009, Funded at ITEA program of European Commission by French Ministry of Industry. Objectives: development and validation of healthcare and wellness platform for physically dependant people (http://www.nuadu.org/).

14 Biswas J, Mokhtari M, Dong JS, Yap P: 'Mild dementia care at home: integrating activity monitoring, user interface plasticity and scenario verification'; In Proc Springer LNCS, Int Conf on smart homes and health Telematics (ICOST'2010). Seoul, June 2010, pp $160-170$. 